\title{
Cinética de Inativação de Inibidores de Tripsina e de Insolubilização de Proteínas de Diferentes Cultivares de Soja
}

\author{
Edna Mayumi Yuahasi Miura ${ }^{1}$, Rui Sérgio dos Santos Ferreira da Silva², \\ Ivone Yurika Mizubuti ${ }^{3 *}$, Elza louko Ida ${ }^{2 *}$
}

\begin{abstract}
RESUMO - A soja (Glycine max (L.) Merrill) é uma das leguminosas mais estudadas, em virtude de sua intensa utilização como fonte de óleo comestível e proteína para alimentação humana e animal. A presença de inibidores de tripsina na soja limita sua utilização, tornando necessário o processamento térmico para melhorar sua qualidade nutricional. Entretanto, o processamento térmico excessivo ou inadequado pode reduzir a qualidade da proteína. Neste trabalho, foram utilizados cultivares de soja BR-36, com atividade normal de inibidores de tripsina, e cultivares BRM 95-5297 e BRM 95-5262, com baixas atividades de inibidores de tripsina. As farinhas com 10 e $16 \%$ de umidade foram aquecidas em banho a $95^{\circ} \mathrm{C}$, com cinco intervalos $(15,25,50,75$ e 100 minutos) de retenção; em seguida, foram determinados os respectivos parâmetros cinéticos de inativação de inibidores de tripsina e de insolubilização de proteínas. A partir da cinética de primeira ordem para inativação de IT e insolubilização de proteínas, observou-se que as constantes de velocidade de reação de inibidores de tripsina aumentaram, com conseqüente redução no tempo de processamento térmico dos cultivares de soja BRM 95-5297 e BRM 95-5262 quando comparados ao cultivar BR-36. O acréscimo de 6\% de umidade nas farinhas também aumentou as constantes de velocidade de inativação de inibidores de tripsina e de insolubilização de proteínas.
\end{abstract}

Palavras-chave: fatores antinutricionais, insolubilidade da proteína, parâmetros cinéticos, tempo de retenção, tratamento térmico

\section{Inactivation Kinetics of Low Activity Trypsin Inhibitors and Protein Insolubilization of Different Soybean Cultivars}

\begin{abstract}
Soybean (Glycine max (L.) Merrill) is one of the most studied vegetables, due to its use as sources of edible oil and protein for human and animal feeding. The presence of trypsin inhibitors in soy grain restrict its utilization and requires heating to improve the nutritional quality. However, excessive heating may reduce the protein quality. Soybean cultivar BR-36 containing normal content of trypsin inhibitors and soybean cultivars (BRM 955297 and BRM 955262 ) with low content of trypsin inhibitors were used. Soybean flour with 10 and $16 \%$ moisture were heated at $95^{\circ} \mathrm{C}$ in five different retention times ( $15,25,50,75$ and 100 $\mathrm{min}$ ) and, then, the respective kinetic inactivation parameters for the trypsin inhibitors and protein insolubilization were estimated. By the first-order reaction for inactivation of trypsin inhibitor and protein insolubilization, the rate constant of trypsin inhibitor reaction incresead, resulting in decreasing heating time of BRM 95-5297 and BRM 95-5262 cultivars when compared to BR-36 cultivar. The increase of $6 \%$ moisture in flours also increased the rate constant of reaction for inactivation of trypsin inhibitors and for protein insolubilization.
\end{abstract}

Key Words: antinutritional factors, heating treatment, kinetics parameters, protein insolubility, retention time

\section{Introdução}

A soja (Glycine max (L.) Merrill) é uma leguminosa importante na alimentação humana e animal, graças às suas características nutricionais, com produção mundial crescente. Entretanto, sua utilização é limitada pela presença de fatores antinutricionais, como os inibidores de tripsina (IT), fatores antinutricionais mais importantes e extensivamente investigados (Pusztai et al., 1991). Os inibidores de tripsina apresentam especificidade de inibir as enzimas proteolíticas e, conseqüentemente, reduzem a digestão protéica dos alimentos e acarretam reduções no ganho de peso e no crescimento dos animais. Também estão relacionados ao aumento no peso do pâncreas de ratos e aves (Liener, 1994).

Cultivares de soja com baixos teores de IT têm sido desenvolvidos com a finalidades de diminuir suas atividades e reduzir os custos no processamento térmico (Domagalski et al., 1992).

\footnotetext{
${ }_{1}^{1}$ Ministério da Agricultura.

2 Docentes do Departamento de Tecnologia de Alimentos e Medicamentos/Centro de Ciências Agrárias/Universidade Estadual de Londrina - UEL, Campus Universitário. Caixa Postal 6001 - CEP: 86051-990. Londrina - Paraná. E.mail: Dra. Elza Iouko Ida elida@uel.br ${ }^{3}$ Docente do Departamento de Zootecnia/CCA/ UEL. Campus Universitário. Caixa Postal 6001 - CEP: 86051-990. Londrina - PR. Brasil. E.mail:mizubuti@uel.br

* Pesquisador bolsista do CNPq.
} 
Comercialmente, existem nos Estados Unidos cultivares de soja com ausência de IT Kunitz (ITK). Porém, há descrições de que, para maximizar a utilização dos componentes nutricionais destes cultivares, ainda seriam necessários tratamentos térmicos menos drásticos que aqueles conferidos à soja com atividade normal de IT (Anderson-Hafermann et al., 1992; Herkelmann et al., 1993; Zhang et al., 1993). No Brasil, a Empresa Brasileira de Pesquisa Agropecuária (EMBRAPA), por intermédio do Centro Nacional de Pesquisa de Soja, no estado do Paraná, desenvolveu cultivares com ausência de ITK (Carrão-Panizzi et al., 1988) e avaliou o rendimento de algumas linhagens avançadas (Carrão-Panizzi, 1997).

Os inibidores de tripsina da soja possuem natureza protéica e estão localizados junto às principais proteínas de armazenamento no corpo protéico do cotilédone (Horisberger et al., 1986) e, geralmente, são fracionados com as proteínas durante o processamento da soja para elaboração de ingredientes alimentares (Anderson \& Wolf, 1995).

Para melhorar o valor nutricional da soja e de seus produtos, há necessidade de tratamentos térmicos para inativar os inibidores de proteases. O tratamento térmico promove a inativação desses inibidores e a desnaturação das globulinas da soja aumentando a susceptibilidade para a proteólise e a disponibilidade dos aminoácidos, melhorando, portanto, a qualidade da proteína para alimentação humana e animal (Snyder \& Kwon, 1987). A inativação térmica de inibidores de tripsina e a insolubilização de proteína de soja foram descritas por Di Pietro \& Liener (1989) e Paula et al. (1995) como reação de primeira ordem. Desse modo, é possível estudar a cinética de inativação das atividades dos inibidores de tripsina e encontrar a melhor condição de inativação de IT e insolubilização de proteínas, com vistas à melhoria da qualidade protéica e à ampliação de sua utilização.

O objetivo nesta investigação foi determinar a cinética de inativação de inibidores de tripsina e de insolubilização de proteínas de diferentes cultivares de soja.

\section{Material e Métodos}

Foram utilizados os cultivares BR-36, com atividade normal de IT, e BRM 95-5297 e BRM 95-5262, com baixas atividades de IT, colhidos em Londrina, PR. Os cultivares BRM 95-5297 e BRM 95-5262 foram provenientes do retro-cruzamento entre o cul- tivar Paraná, com atividades normais de IT, e o PI 157440, com o genótipo de ausência de ITK, desenvolvidos para diminuir os efeitos antinutricionais da soja na alimentação.

O cultivar BR-36 foi selecionado por apresentar atividade normal de IT e produção local. Os cultivares BRM 95-5297 e BRM 95-5262, provenientes do programa de melhoramento genético desenvolvido pela Empresa Brasileira de Pesquisa Agropecuária (Embrapa - Centro Nacional de Pesquisa de Soja), localizada em Londrina, no estado do Paraná, foram utilizados em virtude da baixa atividade de IT e de possíveis reduções de efeitos antinutricionais na alimentação.

Os grãos de soja dos cultivares BR-36, BRM 955297 e BRM 95-5262 foram finamente moídos e classificados em peneira de malha 60 mesh, para obtenção de farinhas integrais com granulometria menor que 0,250 mm. Em seguida, as farinhas integrais foram desengorduradas em aparelho soxhlet com éter de petróleo (AOAC, 1996) durante 24 horas. Com vistas ao processamento térmico das farinhas desengorduradas, foram ajustadas para conterem 10 e $16 \%$ de umidade, para cada cultivar estudado. Objetivando garantir as condições isotérmicas dos tratamentos a $95^{\circ} \mathrm{C}$, cerca de $2 \mathrm{~g}$ de farinhas de soja desengorduradas e pré-aquecidas em banho de óleo a $130^{\circ} \mathrm{C}$ foram colocados em tubos de ensaio com tampa. Os tubos de ensaio foram mantidos a $95^{\circ} \mathrm{C}$ em banho maria, com cinco intervalos de retenção $(\mathrm{t})$, variando de 15 a 100 minutos: $\mathrm{t} 1=15, \mathrm{t} 2=25, \mathrm{t} 3=50$, $\mathrm{t} 4=75$ e $\mathrm{t} 5=100$ minutos. Imediatamente após o tratamento térmico, os tubos de ensaio foram transferidos para um banho de gelo, determinando-se as atividades de IT e a insolubilização de proteínas.

Foram realizados os experimentos 1 e 2 , ambos a $95^{\circ} \mathrm{C}$, utilizando-se farinhas de soja desengorduradas com 10 e $16 \%$ de umidade, respectivamente. Cada um dos três cultivares (tratamentos $(\mathrm{T})$ : $\mathrm{T} 1=\mathrm{BR}-36, \mathrm{~T} 2=$ BRM 95-5297 e T3 = BRM 95-5262) foi submetido a um processamento térmico isostérico (umidade constante) e isotérmico (temperatura constante) por um período de 15 a 100 minutos. Os procedimentos foram realizados em triplicata. Uma abordagem cinética permitiu a modelagem do processo ao longo do tempo e, conseqüentemente, a estimativa dos parâmetros de velocidade.

Os dados obtidos para cada inativação de IT e insolubilização de proteínas foram ajustados à regressão linear (modelo matemático), subsidiada pela respectiva análise de variância. 
As atividades de inibidores de tripsina (AIT) foram determinadas conforme metodologia desenvolvida por Kakade et al. (1974), com modificação no processo de extração, segundo Liu \& Markakis (1989). Para expressão da atividade da tripsina, definiu-se que uma unidade de tripsina (1UT) foi equivalente ao aumento de 0,01 unidades de absorvência a $410 \mathrm{~nm}$, por $10 \mathrm{~mL}$ de solução, sob as condições definidas neste ensaio. Conseqüentemente, uma unidade inibidora de tripsina (1UIT) foi calculada pela diferença entre a mudança na absorvência da tripsina padrão e da amostra, multiplicada por 100. Os níveis de AIT foram determinados pela relação entre UIT e mg de farinha de soja desengordurada. As atividades relativas residuais de IT foram calculadas considerando-se a diferença entre a amostra sem aquecimento, ou seja com $100 \%$ de atividade de IT, e a amostra aquecida.

O índice de insolubilização de proteínas (IIP) indica o grau de eficiência do tratamento térmico sobre a inativação dos inibidores de tripsina e foi calculada por meio da determinação do índice de solubilidade de nitrogênio (ISN), conforme o método descrito por AACC (1990).

Após análise dos dados experimentais de inativação de IT e de insolubilização de proteínas dos respectivos tratamentos, estes foram modelados pela cinética típica de primeira ordem, baseada na descrição de Di Pietro \& Liener (1989). Estes autores preconizaram um modelo cinético simples (log do efeito vs tempo) para modelar (ajustar os dados experimentais) e interpretar a inativação térmica dos inibidores de proteases em soja.

A partir dos resultados cinéticos, foram estimadas as velocidades específicas de reações ( $k$ ) de inativação de IT e de insolubilização de proteínas das farinhas dos três cultivares de soja nos experimentos 1 e 2, com 10 e $16 \%$ de umidade, respectivamente.

\section{Resultados e Discussão}

Constam na Tabela 1 os níveis médios de atividades de IT de farinhas desengorduradas dos cultivares de soja BR-36, BRM 95-5297 e BRM 95-5262. Os níveis de atividades de IT da farinha crua desengordurada do cultivar BR-36, considerada normal, foram de 43,06 UIT/mg de amostra e, para as farinhas de cultivares BRM 95-5297 e BRM 95-5262, com baixas atividades de IT, de 33,06 e 28,15 UIT/mg de amostra, respectivamente.
Sambeth et al. (1967) e Del Valle et al. (1983) descreveram valores de atividades de IT similares para farinha de soja crua. Entretanto, Rackis \& McGhee (1975), Kakade et al. (1974) e Liu \& Markakis (1989) relataram níveis mais elevados, de 87,60 a $184,00 \mathrm{UIT} / \mathrm{mg}$ de amostra. As farinhas desengorduradas dos dois cultivares, BRM 95-5297 e BRM 95-5262, com baixas atividades de IT, apresentaram, respectivamente, 76,77 e 65,37\% de atividade de IT quando comparadas com a farinha desengordurada do cultivar de soja BR-36 com atividade normal de IT. Isso representa, respectivamente, decréscimos de 23,30 e 34,63 \% nos níveis de atividades de IT, em relação ao cultivar BR-36.

Os parâmetros cinéticos (velocidades específicas) foram estimados para se investigar a eficiência dos processos de inativação de IT e de insolubilização de proteínas dos cultivares BR 36, BRM 95-5297 e BRM 95-5262.

A análise de regressão dos dados, expressos como logaritmo (log) das porcentagens das atividades residuais relativas de IT, eos respectivos coeficientes de determinação dos diferentes tratamentos, dos experimentos 1 e 2, estão apresentados na Tabela 2 . Observou-se que a regressão completa foi linear e significativa $(\mathrm{P} \leq 0,01)$ para todos os tratamentos.

As constantes de velocidade de reação de primeira ordem $\left(k, \min ^{-1}\right)$ para a inativação de IT $(k 1)$ estão contidas na Tabela 3.

Para os tratamentos com 10\% de umidade (experimento 1), observou-se que as constantes de velocidade de reação de primeira ordem para as inativações de IT aumentaram aproximadamente quatro vezes entre o cultivar BR-36, com atividades de IT conven-

Tabela 1 - Níveis de inibidores de tripsina (IT)* de farinhas desengorduradas dos cultivares BR-36, BRM 95-5297 e BRM 95-5262 de soja

Table 1 - Trypsin inhibitors (TI) levels of deffated flours of soybean BR-36, BRM 95-5297 and BRM 95-5262

\begin{tabular}{lcc}
\hline $\begin{array}{l}\text { Amostra } \\
\text { Sample }\end{array}$ & $\begin{array}{c}\text { Níveis de IT (UIT/mg de amostra) } \\
\text { TI levels ( TIU/ mg of sample) }\end{array}$ & $\begin{array}{l}\text { UIT (\%) } \\
\text { TIU (\%) }\end{array}$ \\
\hline BR-36 & 43,06 & 100 \\
BRM95-5297 & 33,06 & 76,77 \\
BRM95-5262 & 28,15 & 65,37 \\
\hline
\end{tabular}

* Em triplicata. UIT $=$ Unidades inibidoras de tripsina.

*In three replicates. TIU $=$ Trypsin inhibitors units. 
Tabela 2 - Equações de regressão $(Y)$, expressas em log das porcentagens de atividades residuais relativas de IT dos diferentes tratamentos, e respectivos coeficientes de determinações $\left(R^{2}\right)$

Table 2 - Regression equations $(Y)$ as log of the percentages of trypsin inhibitors relative residual activities of different treatments and respective coefficients of determination $\left(R^{2}\right)$

\begin{tabular}{lcccc}
\hline $\begin{array}{l}\text { Experimento } \\
\text { Experiment }\end{array}$ & $\begin{array}{c}\text { Umidade (\%) } \\
\text { Moisture (\%) }\end{array}$ & $\begin{array}{c}\text { Tratamento (cultivares de soja) } \\
\text { Treatment (soybean cultivars) }\end{array}$ & $\begin{array}{c}\text { Equação } \\
\text { Equation }^{1}\end{array}$ & $\mathrm{R}^{2}$ \\
\hline 1 & 10 & BR-36 & $\hat{\mathrm{Y}}=1,805-0,00053 \mathrm{X}$ & 0,92 \\
& BRM95-5297 & $\hat{\mathrm{Y}}=1,590-0,00186 \mathrm{X}$ & 0,76 \\
& BRM95-5262 & $\hat{\mathrm{Y}}=1,685-0,0023 \mathrm{X}$ & 0,73 \\
& & BR-36 & $\hat{\mathrm{Y}}=1,7524-0,0036 \mathrm{X}$ & 0,99 \\
& \multirow{2}{*}{16} & BRM95-5297 & $\hat{\mathrm{Y}}=1,6680-0,0099 \mathrm{X}$ & 0,98 \\
& & BRM95-5262 & $\hat{\mathrm{Y}}=1,6407-0,0109 \mathrm{X}$ & 0,98 \\
\hline
\end{tabular}

${ }_{1}^{100}$ min e" X e" 15 min.
** Significativo a $1 \%$ de probabilidade.
** Significant at level of $1 \%$ of probability.

Tabela 3 - Constantes de velocidade de reação de primeira ordem para inativação de IT (k1) e insolubilização de proteínas (k2) dos diferentes cultivares

Table 3 - Velocity constants of first order reaction for inactivation of trypsin inhibitors ( $k 1$ ) and for protein insolubilization ( 2 ) of different cultivars

\begin{tabular}{|c|c|c|c|c|}
\hline \multirow[t]{2}{*}{$\begin{array}{l}\text { Experimento } \\
\text { Experiment }\end{array}$} & \multirow[t]{2}{*}{$\begin{array}{l}\text { Umidade (\%) } \\
\text { Moisture (\%) }\end{array}$} & \multirow[t]{2}{*}{$\begin{array}{l}\text { Tratamento (cultivares de soja) } \\
\text { Treatment (Soybean cultivars) }\end{array}$} & \multicolumn{2}{|c|}{$\begin{array}{l}\text { Constantes de velocidades de } \\
\text { reações de primeira ordem } \\
\text { Velocity constants of first order reactions }\end{array}$} \\
\hline & & & $\mathrm{k} 1\left(\min ^{-1}\right)$ & $\mathrm{k} 2\left(\min ^{-1}\right)$ \\
\hline 1 & 10 & $\begin{array}{c}\text { BR-36 } \\
\text { BRM95-5297 } \\
\text { BRM95-5262 }\end{array}$ & $\begin{array}{l}1,15 \times 10^{-3} \\
4,37 \times 10^{-3} \\
5,29 \times 10^{-3}\end{array}$ & $\begin{array}{l}2,71 \times 10^{-3} \\
2,96 \times 10^{-3} \\
4,22 \times 10^{-3}\end{array}$ \\
\hline 2 & 16 & $\begin{array}{c}\text { BR-36 } \\
\text { BRM95-5297 } \\
\text { BRM95-5262 }\end{array}$ & $\begin{array}{l}8,29 \times 10^{-3} \\
22,80 \times 10^{-3} \\
25,10 \times 10^{-3}\end{array}$ & $\begin{array}{l}6,63 \times 10^{-3} \\
8,35 \times 10^{-3} \\
8,18 \times 10^{-3}\end{array}$ \\
\hline
\end{tabular}

cional, e os cultivares BRM 95-5297 e BRM 95-5262, com baixas atividades de IT. Para os tratamentos com $16 \%$ de umidade (experimento 2), este aumento das constantes de velocidade de reação de primeira ordem foi de aproximadamente três vezes.

Analisando-se as velocidades de inativação térmica dos IT, para obtenção de farinhas de soja com mesmas atividade residuais de IT, nos experimentos 1 e 2, verificou-se redução no tempo de processamento térmico para os cultivares com baixas atividades de IT (BRM 95-5297 e BRM 95-5262), em relação ao cultivar BR-36 com atividade normal (Tabela 3). O mesmo fato foi observado por vários investigadores (Anderson-Hafermann,1992; Herkelmann et al., 1993; Zhang et al., 1993), em que os tratamentos térmicos menos drásticos foram aplicados em cultivares com baixas atividades de IT e ausência de ITK.
As farinhas de soja normalmente contêm cerca de $10 \%$ de umidade e, nesta investigação, pode ser verificado que o acréscimo de $6 \%$ de umidade, como aconteceu no experimento 2 em relação ao experimento 1, promoveu aumento da eficiência das inativações térmicas em torno de cinco vezes para cada tratamento, conforme observado nas constantes de velocidades de reações de primeira ordem para as inativações de IT (Tabela 3). Di Pietro \& Liener (1989) observaram que o teor de umidade também influenciou a cinética de inativação de IT de farinha de soja, possivelmente em razão do acréscimo na quantidade de água livre, que elevou as velocidades de insolubilização de proteína e de inativação de IT.

A análise de regressão dos dados, expressos como logaritmo (log) das porcentagens de insolubilização de proteínas medidas pelo ISN, e os 
respectivos coeficientes de determinação dos diferentes tratamentos estão apresentados na Tabela 4.

Observou-se que a regressão completa foi linear e significativa $(\mathrm{P} \leq 0,01)$ para todos os tratamentos.

As constantes de velocidade de reação de primeira ordem $\left(\mathrm{k}, \mathrm{min}^{-1}\right)$ para as insolubilizações de proteínas, medidas pelo ISN $\left(\mathrm{k}^{2}\right)$, para cada tratamento, nos experimentos 1 e 2, estão contidas na Tabela 3. Os valores das constantes de velocidade de insolubilização de proteínas, nos dois experimentos, variaram de 2,71 a $8,35 \times 10^{-3} \mathrm{~min}^{-1}$.

No experimento com $10 \%$ de umidade, observou-se que as constantes de velocidade de reação de insolubilização de proteínas aumentaram em 1,09 e 1,60 vezes para os cultivares BRM 95-5297 e BRM 95-5262, respectivamente, em relação ao cultivar BR 36. Por outro lado, no experimento com $16 \%$ de umidade, esse aumento foi de 1,2 vezes para os dois cultivares, BRM 95-5297 e BRM 95-5262, em relação ao cultivar BR 36.

De modo geral, verificou-se aumento de, no mínimo, duas vezes nas constantes de velocidade de reação de primeira ordem para os tratamentos do experimento 2 , com $16 \%$ de umidade, quando comparados aos tratamentos do experimento 1 , com $10 \%$ de umidade.

Paula et al. (1995) relataram valores de 9,76 x 10-2 $\min ^{-1}$ para as constantes de velocidade de insolubilização de proteínas de farinhas de soja termicamente processadas em autoclave e em condições de excesso de umidade.

Os resultados apresentados nesta investigação confirmam que o excesso de água na amostra eleva as constantes de velocidade de insolubilização de proteínas com conseqüente redução do tempo de processamento térmico. Portanto, nas condições desta investigação, os resultados apresentados obtidos no experimento com $16 \%$ de umidade foram melhores que os do experimento com $10 \%$ de umidade.

Consta na Figura 1 o gráfico da cinética de inativação de IT e, na Figura 2, a cinética de

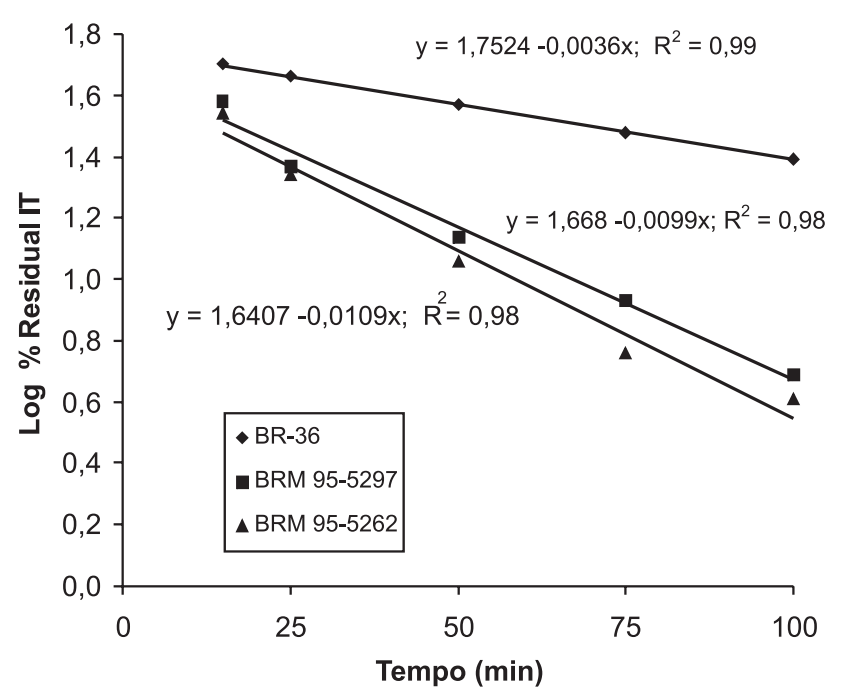

Figura 1 - Cinética de inativação de inibidores de tripsina (IT) de cultivares de soja BR-36, BRM 95-5297 e BRM 95-5262, com 16\% de umidade, em função do tempo de processamento térmico a $95^{\circ} \mathrm{C}$.

Figure 1 - Inactivation kinetics of tripsin inhibitors (TI) of soybean BR-36, BRM 95-5297 and BRM 95-5262 cultivars with $16 \%$ of moisture in function of thermal processing time at $95^{\circ} \mathrm{C}$.

Tabela 4 - Equações de regressão $(Y)$, expressas em log das insolubilizações de proteínas dos diferentes tratamentos, e respectivos coeficientes de determinação $\left(R^{2}\right)$

Table 4 - Regression equations $(Y)$ as log on percentages of protein insolubilization of different treatments and respective coefficients of determination $\left(R^{2}\right)$

\begin{tabular}{lcccc}
\hline $\begin{array}{l}\text { Experimento } \\
\text { Experiment }\end{array}$ & $\begin{array}{c}\text { Umidade (\%) } \\
\text { Moisture (\%) }\end{array}$ & $\begin{array}{c}\text { Tratamento (cultivares de soja) } \\
\text { Treatment (soybean cultivars) }\end{array}$ & $\begin{array}{c}\text { Equação } \\
\text { Equation }^{1}\end{array}$ & $\mathrm{R}^{2}$ \\
\hline 1 & 10 & BR-36 & $\hat{\mathrm{Y}}=1,565-0,0012 \mathrm{X}$ & 0,96 \\
& BRM95-5297 & $\hat{\mathrm{Y}}=1,432-0,0013 \mathrm{X}$ & 0,87 \\
& & BRM95-5262 & $\hat{\mathrm{Y}}=1,522-0,0018 \mathrm{X}$ & 0,84 \\
& \multirow{2}{*}{16} & $\mathrm{Y}=1,6112-0,0028 \mathrm{X}$ & 0,99 \\
& & BR-36 & $\hat{\mathrm{Y}}=1,5473-0,0037 \mathrm{X}$ & 0,98 \\
& BRM95-5297 & $\hat{\mathrm{Y}}=1,5288-0,0035 \mathrm{X}$ & 0,98 \\
\hline
\end{tabular}

$100 \min$ e" $X$ e" $15 \min$.

** Significativo a $1 \%$ de probabilidade.

** Significant at level of $1 \%$ of probability. 


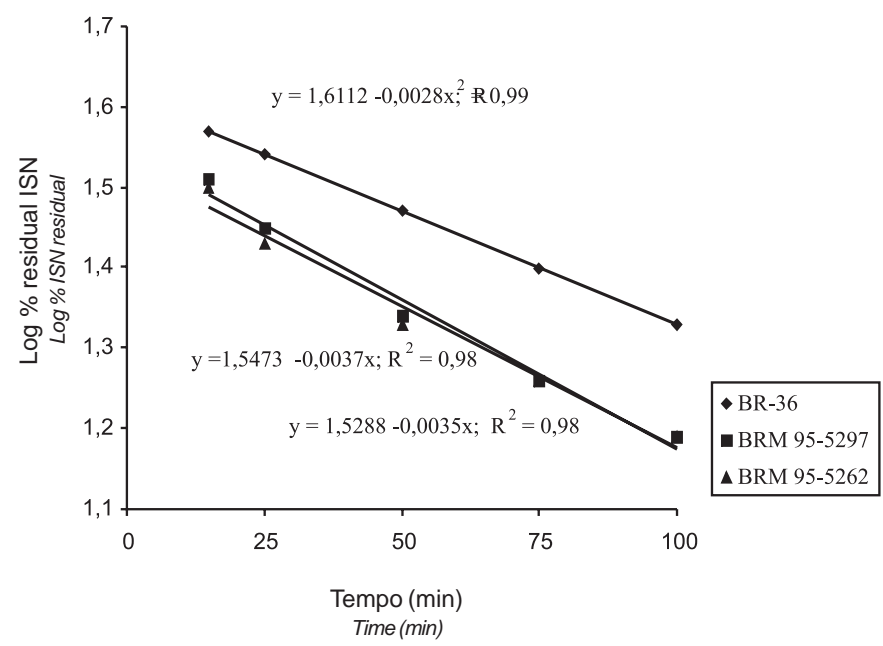

Figura 2 - Cinética de insolubilização de proteínas medidas em ISN, dos cultivares BR-36, BRM 95-5297 e BRM 95-5262 de soja, com $16 \%$ de umidade, em função do tempo de processamento térmico a $95^{\circ} \mathrm{C}$

Figure 2 - Insolubilization kinetics of protein measured in ISN of BR-36, BRM 95-5297 and BRM 95-5262 soybean cultivars with $16 \%$ moisture, in function of thermal processing time at $95^{\circ} \mathrm{C}$.

insolubilização de proteínas (IIP medidas em ISN) no processamento térmico dos cultivares BR-36, BRM 95-5297 e BRM 95-5262, com 16\% de umidade, em função do tempo de processamento, em minutos.

A cinética de inativação de IT e a de insolubilização de proteínas apresentaram efeitos lineares. Entretanto, houve menor inclinação das retas para a cinética de insolubilização de proteínas (Figura 2) que para a de inativação de IT (Figura 1). Ou seja, as inativações de IT foram mais rápidas que as insolubilizações de proteínas. Rackis \& McGhee (1975) também observaram, em farinhas de soja, que a diminuição das solubilidades de proteínas, estimadas pelo ISN, foi mais lenta que as respectivas inativações de IT.

\section{Conclusões}

As constantes de velocidade de reação para cinética de primeira ordem $\left(\mathrm{min}^{-1}\right)$ de inibidores de tripsina dos cultivares de soja BRM 95-5297 e BRM 95-5262 (com baixas atividades de IT) foram maiores que para o cultivar BR-36, reduzindo o tempo de processamento térmico.

O acréscimo de $6 \%$ de umidade na farinha de soja elevou as constantes de velocidade de reação de inativação de IT e o índice de insolubilização de proteínas.
As constantes de velocidade de reação de primeira ordem para as inativações de IT comprovaram que 16\% de umidade na soja promoveu aumento da eficiência de inativações térmicas.

\section{Literatura Citada}

AMERICAN ASSOCIATION OF CEREAL CHEMISTS AACC. Approved methods. 8.ed. Minnesota, 1990. p.46-23. ASSOCIATION OF OFFICIAL ANALYTICAL CHEMISTS AOAC. Official methods of analysis. Kenneth Helrich. Arlington, Virginia. v.2. 1996.1298p.

ANDERSON-HAFERMANN, J.C.; ZHANG, Y.; PARSONS, C.M. Effect of heating on nutritional quality of conventional and Kunitz trypsin inhibitor-free soybeans. Poultry Science, v.71, p.1700-1709, 1992.

ANDERSON, R.L.; WOLF, W.J. Compositional changes in tripsyn inhibitors, phytic acid, saponins and isofhavones related to soybean processing. Journal of nutrition, v.125, p.581S-588S, 1995.

CARRÃO-PANIZZI, M.C. Desenvolvimento de germoplasma de soja com características adequadas para o consumo humano "in natura" e para a indústria de alimentos (04.0.94.321.-07). Resultados de pesquisa de soja 1993/1995. Londrina: Embrapa-soja, p.14-16, 1997.

CARRÃO-PANIZZI, M.C.; KIIHL, R.A.S., TOLEDO, J.F.F. Desenvolvimento de cultivares para consumo humano “in natura” e para a indústria de alimentos. Resultados de pesquisa de soja 1986/87. Londrina: Embrapa-soja, 1988. p.256-257.

DEL VALLE, F.R.; PICO, M.L.; CAMACHO, J.L. et al. Effect of processing parameters on trypsin inhibitor and lectin contents of tortillas from whole raw corn-soybean mixtures. Journal of Food Science, v.48, p.246-252, 1983.

DI PIETRO, C.M.; LIENER, I.E. Heat inactivation of the Kunitz and Bowman-Birk soybean protease inhibitors. Journal of Agricultural Food Chemistry, v.37, p.39-44, 1989.

DOMAGALSKI, J.M.; KOLLIPARA, K.P.; BATES, A.H. et al. Nulls for the major soybean Bowman-Birk protease inhibitor in the genus Glycine. Crop Science, v.32, p.1502-1505, 1992.

HERKELMANN, K.L.; CROMWELL, G.L.; CANTOR, A.H. et al. Effects of heat treatment on the nutritional value of conventional and low trypsin inhibitor soybeans for chicks. Poultry Science, v.72, p.1359-1369, 1993.

HORISBERGER, M.; CLERC, M.F.; PAHUD, J.J. Ultrastrutural localization of glycinin and â-conglycinin in Glycine max (soybean) cv. Maple arrow by the immunogold method. Histochemistry, v.85, p.191-194,1986.

KAKADE, M.L.; RACKIS, J.J.; McGHEE, J.E. et al. Determination of trypsin inhibitor activity of soy products: a collaborative analysis of an improved procedure. Cereal Chemistry, v.51, p.376-382, 1974.

LIENER, I.E. Implications of antinutritional components in soybeans foods. CRC Critical Review in Food Science and Nutrition, v.34, n.1, p.31-67, 1994.

LIU, K.; MARKAKIS, P. An improved colorimetric method for determining antitryptic activity in soybean products. Cereal Chemistry, v.66, p.415-422, 1989.

PAULA, A.C.O.; CARVALHO JR., I.C.; CABRAL, L.C. et al. Estudo cinético da inativação térmica da lipoxigenase e da 
insolubilização protéica na soja. Ciência e Tecnologia de Alimentos, v.15, p.262-267, 1995.

PUSZTAI, A.; WATT, W.B.; STEWART, J.C. A comprehensive scheme for the isolation of trypsin inhibitors and the aglutinin from soybean seeds. Journal of Agricultural and Food Chemistry, v.39, p.862-866, 1991.

RACKIS, J.J.; McGHEE, J.E. Biological threshold levels of soybean trypsin inhibitors by rat biossay. Cereal Chemistry, v.52, p.85-92, 1975.

SAMBETH, W.; NESHEIM, M.C.; SERAFIN, J.A. Separation of soybean whey into fractions with different biological activities for chicks and rats. Journal of Nutrition, v.92, p.479-490, 1967.
SNYDER, H.E.; KWON, T.W. Soybean utilization. New York: Van Nostrand Reinhold Company, 1987. 346p.

ZHANG, Y.; PARSONS, C.M.; WEINGARTNER, K.E. et al. Effects of extrusion and expelling on the nutritional quality of conventional and Kunitz trypsin inhibitor-free soybeans. Poultry Science, v.72, p.2299-2308, 1993.

Recebido em: 07/02/04

Aceito em: 12/04/05 